\title{
Liderazgo y su relación con la creatividad en estudiantes universitarios
}

\section{Leadership and its Relationship with Creativity in University Students}

Raúl Chávez Silva* https://orcid.org/0000-0002-9619-1766

Lorena Gallegos Vela** https://orcid.org/0000-0001-5707-6960

Enma Carrasco Campos***https://orcid.org/0000-0003-3564-8053

Manuel Hernández Félix**** https://orcid.org/0000-0002-4952-6105

http://dx.doi.org/10.21503/lex.v19i27.2261

* Magíster en Educación. Universidad Alas Peruanas. Perú.

Correo electrónico: rchsilva@yahoo.com

** Doctora en Educación. Universidad Alas Peruanas. Perú.

Correo electrónico: lorgave10@outlook.com; 1_gallegos@uap.edu.pe

*** Doctora en Educación. Universidad Alas Peruanas. Perú.

Correo electrónico: enma_carrasco@hotmail.com; e_carrasco@uap.edu.pe

**** Magíster en Educación. Universidad Alas Peruanas. Perú.

Correo electrónico: manupicchuperu@gmail.com; m_hernandez_f@doc.uap.edu.pe

El presente artículo de investigación es producto del proyecto sobre Liderazgo y su relación con la creatividad en estudiantes universitarios, financiada por el Vicerrectorado de Investigación, Innovación y Emprendimiento de la Universidad Alas Peruanas en el año 2019.

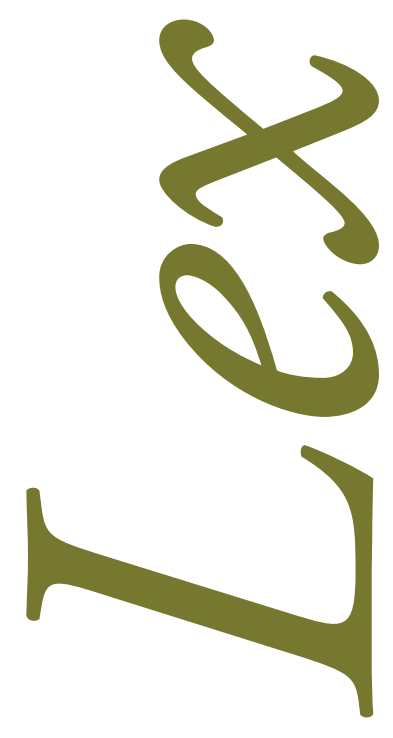




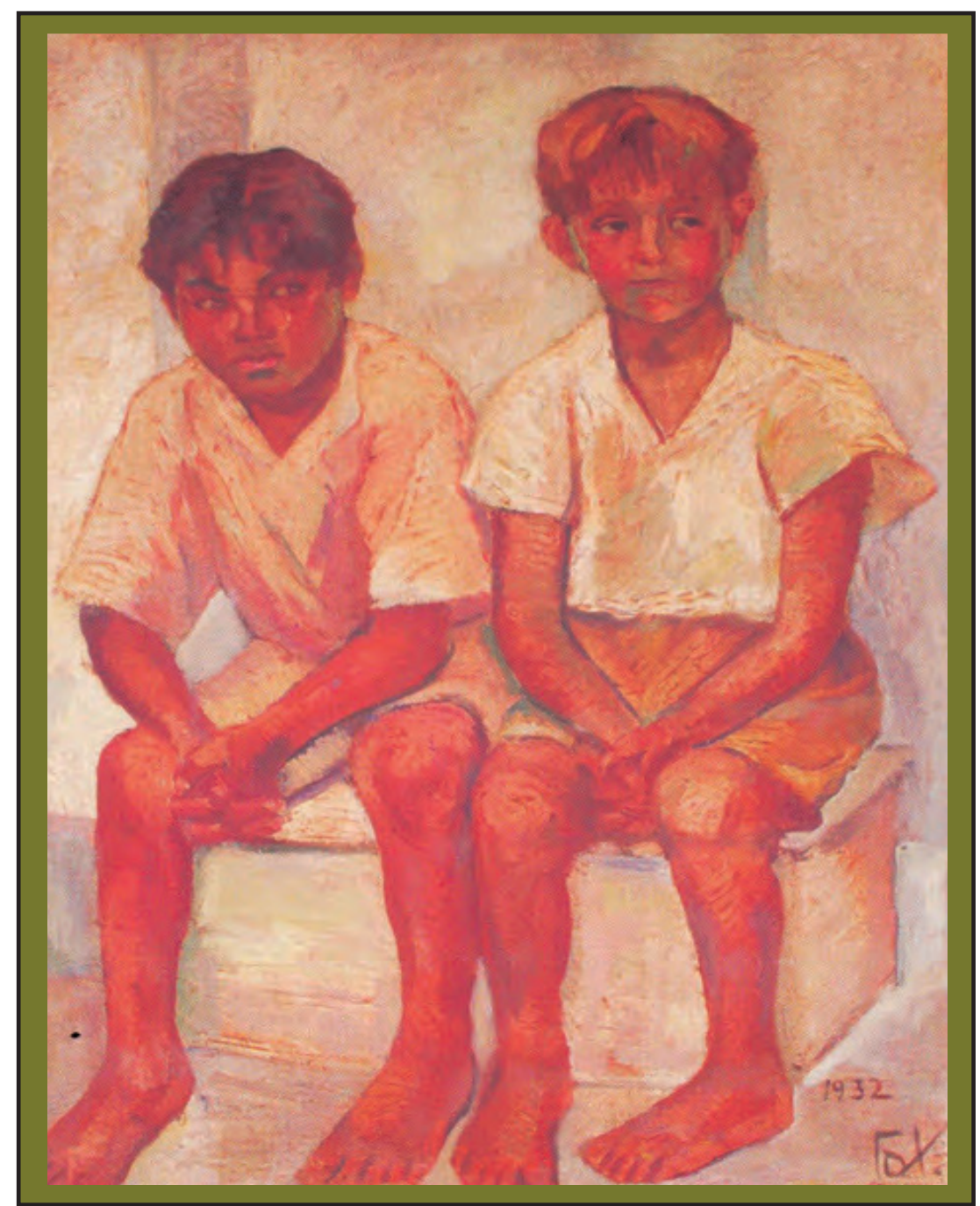

Los paniches. Carlota Carvallo pintora peruana del indigenismo ( Lima 1909-1980). 


\title{
RESUMEN
}

Numerosas investigaciones sobre el liderazgo y la creatividad en las organizaciones sociales señalan una relación entre ambas; sin embargo, queda por ampliar dicha explicación en grupos que se preparan para ejercer la docencia en la educación básica. Nuestro objetivo constituyó en comprobar la relación en un contexto académico, utilizando instrumentos desarrollados por estudiosos como Fischman (liderazgo) y Dadamia (creatividad). Se realizó un estudio en una muestra estratificada de 147 estudiantes universitarios en la cual el $97 \%$ fue de sexo femenino. El resultado estadístico no paramétrico Rho de Spearman muestra un Coeficiente de correlación débil de ,237 con una significancia de, 001 (bilateral).

Palabras clave: creatividad, innovación educativa, liderazgo.

\begin{abstract}
Numerous research on leadership and creativity in social organizations points to a relationship between them; however, this explanation remains to be extended in groups that are preparing to teach in basic education. Our goal was to verify the relationship in an academic context, using instruments developed by scholars such as Fischman (leadership) and Dadamia (creativity). A study was conducted on a stratified sample of 148 university students in which $97 \%$ were female. Spearman's Rho non-parametric statistical result shows a weak correlation coefficient of, 237 with a significance of, 001 (bilateral).
\end{abstract}

Keywords: creativity, educational innovation, leadership. 


\section{INTRODUCCIÓN}

Desde hace algunas décadas, existe en el mundo la tendencia a considerar en el currículo de las instituciones formadoras de docentes para la educación básica, al liderazgo y la creatividad entre las "habilidades blandas" que deben poseer los nuevos educadores. Sin embargo, los diseñadores de los programas curriculares de maestros ignoran la demanda del mercado y el impacto que tienen el liderazgo y la creatividad en el desarrollo de las capacidades que requiere el hombre para adaptarse y producir más y mejor en la sociedad del conocimiento y la imaginación. Este desdén por el liderazgo y la creatividad impacta negativamente en la formación de los estudiantes de educación básica, quienes continúan reproduciendo la cultura que aprenden de sus maestros y del entorno. Frente a este problema, las organizaciones responsables de diseñar la carrera docente tampoco asumen una actitud de cambio e ignoran el valor que ha cobrado el pensamiento creativo y el liderazgo innovador en la instrucción como en la dirección de organizaciones. Una consecuencia inevitable es que la mayoría de los egresados de la escuela básica, no son formados para incorporarse al sistema laboral para contribuir en la generación de riqueza mediante la producción de bienes y servicios innovadores de acogida en el mercado. Con respecto al problema, en el Perú como en muchos países, Drucker , afirma que el líder para adelantarse al futuro tiene que practicar "asiduamente dos destrezas orientadas al futuro: la observación de las tendencias y la imaginación" 1 . El mismo estudioso, observa que "la falta de liderazgo es evidente en toda la sociedad" ${ }^{2}$. Esta cuestión no se limita a los países del tercer mundo, las organizaciones del siglo XXI adolecen de líderes equipados para hacer frente a la complejidad y las nuevas reglas del mercado. Ser líder implica, entre otras cosas, ser creativo, pero la educación no responde a las exigencias sociales, es decir, se carece de docentes líderes de visión creativa y en condiciones de revolucionar la educación.

Asimismo, la investigación sobre este tema plantea interrogantes como: ¿el oficio de enseñar requie-

1. P. Drucker, El líder del futuro, (Argentina: Deusto S.A., 1997), 157.

2. Ibídem, 191. 
re que los docentes sean líderes creativos?, ¿es posible combinar ambas cualidades en un solo sujeto?, ¿qué ventajas tendría el líder creativo frente a los demás en la tarea de la gestión o de la enseñanza? El fenómeno del liderazgo vinculado a la creatividad es inevitable estudiarlo en los tiempos actuales, pues es una ventaja competitiva en las organizaciones presionadas por la dinámica del cambio y la innovación. Según Molinar y Velázquez: "Las habilidades se pueden aprender y desarrollar en la práctica. El docente, como líder, motiva a los estudiantes a crecer y desarrollar habilidades que les encamina a ser líderes"3. El profesor recién egresado del sistema educativo tiene que saber gestionar los recursos pedagógicos de la escuela, para producir nuevos conocimientos, habilidades y actitudes en los estudiantes, mediante el uso de fortalezas para visionar el futuro, identificar y resolver problemas de una manera original, tomar decisiones rápidas y favorables en beneficio del desempeño integral de los estudiantes.

Los estudios sobre el liderazgo y la creatividad se han intensificado en los últimos años, debido al auge de las teorías que sostienen que el talento humano es el factor más importante en el desarrollo de las organizaciones sociales cuando sus individuos exhiben cualidades de liderazgo y creatividad, que han sido estimuladas tempranamente desde la escuela. Al respecto, Dadamia, al referirse a la creatividad, sostiene que "Los movimientos pedagógicos de vanguardia, las escuelas nuevas, la escuela activa, en todos los países, afirman que la creatividad es una aptitud que se desarrolla con la práctica; y son consecuentes con esta afirmación al dar cabida dentro de sus programaciones a experiencias y actividades que

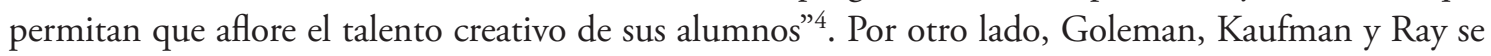
interesaron en explorar el mundo en búsqueda del "espíritu creativo", y dan cuenta en extraordinarios relatos de algunas escuelas que han impregnado la creatividad en el currículo de una manera concreta y con resultados fabulosos, como las escuelas Reggio-Emilia de Italia y Key School de Indianápolis ${ }^{5}$. El liderazgo y la creatividad son necesarias en los maestros de aula y directores para gestionar el cambio productivamente. Chiavenato, al referirse al cambio, sostiene que las personas en las organizaciones produzcan más y mejor, pero "para promover cambios internos el mayor desafío es cambiar la cabeza de las personas, tornarlas más eficientes y eficaces, más integradas, más productivas e imaginativas”. Para conseguirlo, hay que formar líderes capaces de mirar la realidad de manera diferente y creativa. $\mathrm{Y}$ es tarea de la educación crear lideres imaginativos desde el aula, como lo afirma Gardner al sostener que "importantes dimensiones de la creatividad adulta tienen sus raíces en la infancia del creador"7, quien también considera que el contexto social influye en la creatividad de los niños, lo cual incluye a sus familiares y, en forma especial, los que están implicados en su educación como sus maestros ${ }^{8}$. Sin embargo, la educación ignora las características del desarrollo cognitivo del niño quien, por ser miem-

3. M. Molinar y L.Velásquez, Liderazgo en la labor docente, (México: Trillas, 2015), 31.

4. O. Dadamia, Educación y creatividad, (Argentina: Magisterio del Río de la Plata, 2001), 22.

5. D. Goleman, P. Kaufman y M. Ray, El espíritu creativo, (Argentina: Vergara, 2000), 103 - 114.

6. I. Chiavenato, Construcción de talentos, (Colombia: Mc Graw Hill, 2004), 8.

7. H. Gardner, Mentes creativas, (España: Paidos, 1998), 47.

8. Ibídem, 47. 
bro de la especie humana, es curioso por naturaleza. Al respecto, el niño al explorar su entorno tiene la oportunidad de descubrir las leyes que rigen la realidad del mundo físico, social y del mismo, y que estos "procesos de descubrimiento se convierten en modelos de las conductas exploratorias posteriores, incluidos los esfuerzos por investigar fenómenos nunca conceptualizados" ${ }^{\text {" }}$.

No cabe duda de que los maestros tradicionales no tienen capacidad para mirar la realidad de una manera diferente a los maestros creativos, para ello se requiere haber desarrollado habilidades de flexibilidad para abandonar los esquemas fijos que heredamos de la cultura tradicional y conservadora. Ser líder implica, entre otras cosas, ser creativo. Ponti, al definir las características de las empresas creativas, afirma que ellas deben estar en manos de personas que de verdad fomenten en el personal la imaginación creativa ${ }^{10}$. Asimismo, hay quienes como Buzan, Dottino e Israel consideran que "Los líderes inteligentes son preparadores. Tienen que animar a su personal a rendir al máximo, hasta un límite que nunca había pensado que podían alcanzar. Deben comunicar su visión a su personal para conseguir que se convierta en realidad"11.

Sin embargo, los estudios sobre el liderazgo y la creatividad dan mayor énfasis a la gestión de las organizaciones económicas y en las educativas a la gestión del director. A lo sumo, durante la carrera de educación donde se hizo el estudio, el currículo contempla talleres de liderazgo o creatividad en el segmento de las asignaturas electivas, de cuatro horas semanales cada uno. Lo anterior grafica de cuerpo entero la existencia de un problema para abordar inteligentemente, Por lo tanto, la investigación es una oportunidad de acometer la empresa de buscar nexos entre el liderazgo y la creatividad en los estudiantes de las carreras de educación inicial y primaria, cuyos resultados pueden servir para fundamentar cambios curriculares y determinar el perfil del egresado de la Escuela Profesional de Educación de la Universidad Alas Peruanas.

Desde el punto de vista teórico, la investigación es importante por la urgencia de verificar la solidez de las ideas de los pensadores de la creatividad y del liderazgo, quienes afirman que ella son dos variables que se encuentran íntimamente relacionadas. De ser así, el resultado nos permite tener un mejor conocimiento de ellas y plantear nuevas interrogantes sobre su desenvolvimiento en la experiencia educativa de los grupos de estudiantes de la carrera de educación.

Desde la perspectiva práctica, la investigación está plenamente justificada, porque la época que vivimos constituye el momento ideal para incorporar la creatividad y el liderazgo en los programas educativos de formación docente como competencias transversales del currículo.

9. Ibídem, 49.

10. F. Ponti, La empresa creativa, (Barcelona: Granica, 2001), 137.

11. T. Buzan, T. Dottino y R. Israel, La inteligencia del líder, (España: Deusto, S.A., 1999), 138. 


\section{MATERIAL Y MÉTODOS}

El estudio se realizó en una muestra probabilística por estratos de 147 personas, 143 mujeres y 4 hombres. Los participantes eran estudiantes de los 10 ciclos académicos del semestre 2019 - I de la Escuela de Educación de la Facultad de Ciencias Empresariales y Educación de la Universidad Alas Peruanas, que lo hicieron en forma voluntaria.

Recogimos los datos el 28 de mayo del 2019 con el apoyo de los docentes, capacitados para administrar la aplicación de los cuestionaros, en dos turnos: del 1er. al 5to. ciclo en la mañana, y del 6to. al 10mo. por la tarde. Primero se evaluó el liderazgo a través de 124 ítems en los que se les preguntaba aspectos como control del ego (20 ítems), equilibrio (21 ítems), desapego (20 ítems), responsabilidad (20 ítems), estudio en equipo (18 ítems) y destrezas directivas (25 ítems). Las categorías de respuesta fueron con una escala tipo Likert de 1 (muy bajo liderazgo) a 5 (muy alto liderazgo). Este cuestionario fue adaptado del original de Fischman (2000), elaborado por él para medir el liderazgo empresarial. La creatividad se midió con un cuestionario de 20 ítems: cuatro para cada una de las siguientes dimensiones: cognición, concepción, combustión, consumación y comunicación, creado por Dadamia (2001) y con variaciones de 1 (baja creatividad) a 3 (alta creatividad). Las repuestas se organizaron en una base de datos sobre la cual se aplicó el análisis descriptivo y el bivariable correlacional Rho de Spearman mediante el software SPSS versión 23 español.

Figura 1: Estuadiantes responden cuestionario de Liderazgo y Creatividad

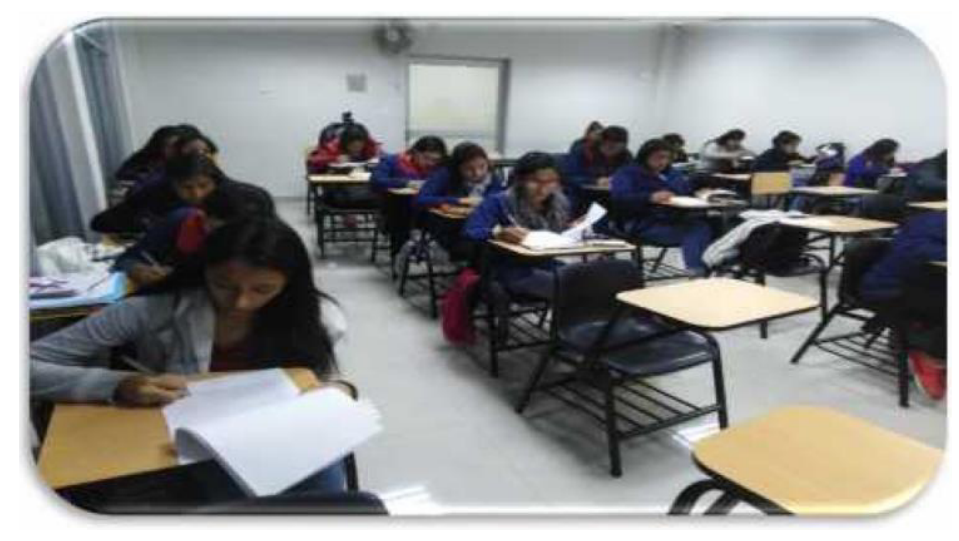




\section{RESULTADOS}

Tabla $\mathrm{N}^{\circ}$ 1: Niveles de liderazgo

\begin{tabular}{|c|c|c|c|c|c|}
\hline & & Frecuencia & Porcentaje & $\begin{array}{c}\text { Porcentaje } \\
\text { válido }\end{array}$ & $\begin{array}{l}\text { Porcentaje } \\
\text { acumulado }\end{array}$ \\
\hline \multirow[t]{6}{*}{ Válido } & Muy bajo liderazgo & 0 &, 0 & 0 &, 0 \\
\hline & Bajo liderazgo & 4 & 2,7 & 2,7 & 2,7 \\
\hline & Moderado liderazgo & 96 & 65,3 & 65,3 & 68,0 \\
\hline & Alto liderazgo & 47 & 32,0 & 32,0 & 100,0 \\
\hline & Muy alto liderazgo & 0 &, 0 &, 0 & \\
\hline & Total & 147 & 100,0 & 100,0 & \\
\hline
\end{tabular}

Fuente: Base de datos

Tabla $\mathrm{N}^{\circ}$ 2: Niveles de creatividad

\begin{tabular}{|c|c|c|c|c|c|}
\hline & & Frecuencia & Porcentaje & $\begin{array}{c}\text { Porcentaje } \\
\text { válido }\end{array}$ & $\begin{array}{l}\text { Porcentaje } \\
\text { acumulado }\end{array}$ \\
\hline \multirow[t]{4}{*}{ Válido } & Baja creatividad & 0 & , 0 &, 0 &, 0 \\
\hline & Mediana creatividad & 35 & 23.8 & 23.8 & 23.8 \\
\hline & Alta creatividad & 112 & 76,2 & 76,2 & 100,0 \\
\hline & Total & 147 & 1000 & 1000 & \\
\hline
\end{tabular}

Fuente: Base de datos

Tabla $N^{\circ}$ 3: Niveles de creatividad

\begin{tabular}{|c|c|c|c|c|}
\hline & & & LIDERAZGO & CREATIVIDAD \\
\hline \multirow[t]{5}{*}{ Rho de Spearman } & \multirow[t]{3}{*}{ LIDERAZGO } & Coeficiente de correlación & 1.000 & $237^{*}$ \\
\hline & & Sig. (bilateral) & & .004 \\
\hline & & $\mathrm{N}$ & 147 & 147 \\
\hline & \multirow[t]{2}{*}{ CREATIVIDAD } & Coeficiente de correlación &, $237^{* \prime}$ & 1,000 \\
\hline & & Sig. (bilateral) & 004 & \\
\hline & & $\mathrm{N}$ & 147 & 147 \\
\hline
\end{tabular}

\section{DISCUSIÓN}

En nuestro estudio se investigó los niveles y la relación entre el liderazgo y la creatividad. El resultado refleja el predominio de niveles de liderazgo moderado y alta creatividad y una débil relación entre ambas, lo cual permite mantener la hipótesis y abre la posibilidad de poder extender la investigación a estudios experimentales en instituciones que consideren las competencias de liderazgo y creatividad en sus planes de estudio. 
En la literatura sobre este tema, predominan los estudios centrados en el liderazgo creativo de gerentes de empresas y directores de instituciones educativas, y muy pocos dedicados a los estudiantes de la carrera de educación, como es nuestro caso, de ahí el valor de las ideas de pensadores como Molinar y Velázquez, Drucker, Chiavenato, entre otros, para quienes todas las personas somos capaces de aprender a ser líderes creativos.

Un aspecto novedoso de nuestra investigación es haber utilizado como dimensiones del liderazgo las características que señala Fischman, a partir de la idea del conocimiento de sí mismo y el hecho de que la tarea del líder es formar otros líderes así como las fases del proceso creador en la educación de Dadamia, lo cual abre muchas puertas a nuestra investigación: intentar explicar el peso de cada una de esas dimensiones en la relación con la creatividad; observar si los niveles y la relación mejoran al pasar de un grado académico a otro de mayor nivel en la ruta hacia el final de la carrera; saber si el género de los estudiantes marca diferencias en la intensidad de la relación; determinar si la inclusión en el plan de estudios de contenidos de liderazgo y creatividad impacta favorablemente en el desarrollo del liderazgo creativo; y hacer estudios comparativos con estudiantes de otras escuelas de la universidad.

\section{CONCLUSIONES}

1. La prueba estadística con Rho de Spearman arrojó un Coeficiente de correlación débil (,237) con una probabilidad de $4 \%$ de error, siendo significativa en el nivel ,01 (bilateral), lo cual permite apoyar la hipótesis al encontrarse evidencias empíricas, aunque débiles, del nexo entre ambas variables.

2. En el grupo, 96 estudiantes (65,3\%) reflejan ejercer un liderazgo medio o moderado y 47 $(32 \%)$ muestra un liderazgo alto. Estos niveles son significativos debido a que el plan de estudios no considera al liderazgo como una competencia básica en la carrera docente.

3. En la creatividad los porcentajes alcanzados por el grupo reflejan niveles de alta creatividad $(76,2)$, es decir, tres veces más que los ubicados en el nivel de creatividad media: 23,8 \%. Al igual que en el liderazgo, los estudiantes muestran ser creativos, a pesar de no ser estimulados hacia ella por el sistema pedagógico de la Escuela de Educación. 


\section{REFERENCIAS}

- $\quad$ Buzan, T., Dottino, T. e Israel, R. La inteligencia del lider. España: Deusto S.A., 1999.

- Chiavenato, I. Construcción de talentos. Colombia: Mc Graw Hill., 2004.

- Dadamia, O. Educación y creatividad. Argentina: Magisterio del Río de la Plata, 2001.

- $\quad$ Drucker, P. El lider del futuro. Argentina: Deusto S.A. 1997.

- Fischman, D. El espejo del líder. Perú: El Comercio, 2000.

- Gardner, H. Mentes creativas. España: Paidos, 1998.

- Goleman, D., Kaufman, P. y Ray, M. El espiritu creativo. Argentina: Vergara, 2000.

- Molinar, M. y Velásquez, L. Liderazgo en la labor docente. México: Trillas, 2015.

- $\quad$ Ponti, F. La empresa creativa. Barcelona: Granica, 2001.

RECIBIDO: $12 / 10 / 2020$

APROBADO: $10 / 11 / 2020$ 